\begin{tabular}{lll}
\hline No 75 & Juillet & 1888
\end{tabular}

\title{
COMITÉ INTERNATIONAL
}

\section{ACTIVITÉ MARITIME DES SOCIÉTÉS DE LA CROIX-HOUGE}

Soixante et onzième circulaire aux Comités centraux.

Gent̀ve, le 18 Juin 1888.

\section{Messieurs,}

En conformité d'une décision prise, le 27 seplembre 1887, par la Conférence internationale de Carlsruhe, nous nous faisons un devoir de porter devant vous la question de l'activité maritime des Sociétés de la Croix-Rouge.

Qu'il nous soit permis de vous faire remarquer, avant tout, que la résolution à laquelle nous nous référons n'a pas élé rédigée au moment du vote et ne se trouve pas formulée avec précision dans le compte rendu de la Conférence. Ce dernier document dit seulement que « les propositions de MM. Hyades et Hass ont été adoptées » et, pour en connaître la teneur, il faut relire la délibération au cours de laquelle elles se sont produites. On y voit que, tandis que M. Hass ne demandait que l'ajournement du débat, M. Hyades désirait de plus que la question fùt prochainement étudiée par le Comité international, pour faire de sa part l'objet d'un rapport ultérieur. Il spécifiait aussi que le Comité international préparerait ce rapport «avec les délégués des puissances, 》 et le «communiquerait à tous les gouvernements qui ont adhéré à la Convention de Genève.»)

Or, nous croyons que ces derniers desiderata, quoique ratifiés implicitement par la Conférence, ne doivent pas être pris à la lettre, et que c'est par pure inadvertance que les Comités centraux n'ont pas étẻ mentionnés comme devant coopérer, avec le Comité international, à l'enquête dont il s'agit. Nous ne saurions admettre, en eflet, une procédure suivant laquelle nous nous présenterions devant la prochaine conférence comme les interprètes des gouvernements, touchantl'activité maritime des Sociétés de la Croix. 
Rouge, sans avoir pris l'avis préalable de ces Sociétés elles-mêmes.

Du reste, hâtons-nous de le dire, M. le Dr Hyades est pleinement d'accord avec nous sur ce poiut. Il résulte de la correspondance que nous avons échangée avec lui, que nous agirions conformément à sa pensée, si nous demandions à chaque Comité central de formuler un projet détaillé, de le faire approuver éventuellement par l'autorité compétente de son pays et de nous l'envoyer ; puis si, à l'aide de cet ensemble d'informations, nous présentions à la future conférence un rapport général, dans lequel il serait, de la sorte, tenu compte aussi bien de l'opinion des Sociétés de la Groix-Rouge que de celle des gouvernements.

Telle est, par conséquent, la ligne de conduite que nous nous proposons de suivre, et c'est pourquoi nous venons; aujourd'hui, réclamer de tous les Comités centraux, mais plus spécialement de ceux des puissances maritimes, leur indispensable concours, pour nous acquitter du mandat que la Conférence de Carlsruhe nous a fait l'honneur de nous confier.

Vous n'ignorez pas, Messieurs, que la matière que nous proposons à volre examen a déjà fait l'objet d'un débat contradictoire à la Conférence de Berlin, en 1869, et que tont un programme d'action fut adopté par cette assemblée. Vous savez aussi que ce plan est demenré une lettre morte, par suite de la non-ratification, par les gouvernements, du Projet d'articles additionnels à la Convention de Genève. Mais cela n'a pas empêché la Conférence de Carlsruhe de se montrer favorable à une nouvelle étude du sujet, motivée par les profonds changements qui se sont opérés, depuis 1869 , dans les conditions de la guerre maritime, car ces changements autorisent à considérer comme surannées les dispositions arrêtées il y a dix-neuf ans par les Sociétés de secours.

Des motifs de haute convenance ont ẹmpêché la Conférence de Carlsruhe d'émettre un vœu en faveur de l'adoption définitive on de la revision des Articles additionnels de 1868, mais il est présumable que les gouvernements en prendront eux-mêmes l'initiative, lorsqu'ils verront les Sociétés de la Croix-Rouge, dont l'intervention est aujourd'hui si appréciée dans les guerres terrestres, prêtes à les assister, à celte condition, en cas de guerre maritime.

Nons espérons, Messieurs, que, vu l'importance du travail pour lequel nous faisons appel à votre zèle, vous vondrez bien vous en 
occuper dès à présent. Comme nous le disions plus hant, il s'agirait, pour chaque Comité central, de tracer, d'accord avec son gollvernement, un programme préparaloire pour son activité navale et de nous faire parvenir ce document, de telle sorte que, lorsque la future conférence abordera à son tour cette étude, nous puissions en mettre sous ses yeux tous les éléments.

L'enquête confiée au Comité international nous semble devoir porter sur trois points, qui épuisent la matière :

1. Quel rỏle les Sociétés de secours poinraient-elles remplir utilement dans une guerre maritime?

9. De quel matériel ces Sociétés auraient-elles besoin pour cela, et comment pourraient-elles se le procurer?

3. Quel personnel leur faudrait-il, et ou pourraient-elles le recruter?

Pensant qu'il vous sera agréable d'a voir pour guide, dans cetle recherche, les idées qui ont prévalu à la Conférence de Berlin, nous les rappelons ci-après, dans un ordre qui répond à celui des trois questions que nons venons de poser.

Nous ne fixons aucun délai pour l'envoi de votre réponse à la présente circulaire, car nous comprenons qu'elle pourra subir des retards indépendants de votre volonté. Nous nous bornons à vous prier de ne pas perdre de vue notre requête et d'y satisfaire aussitòt que possible.

Agréez, Messieurs, l'assurance de notre considération dislinguée.

Pour le Comité International de la Croix-Rouge:

\section{Le Président,}

Le Secrétaire,

G. Moynier.

E. ODIER.

\section{L'ACTIVITÉ MARITIME DES SOCIÉTÉS DE LA CROIX-ROUGE d'après la Conférence internationale de Berlin}

(Avril 1869)

Nota. - Les numéros placés entre parenthèses renvoient aux alinéas du textè officiel des Résolutions príses à Berlin, Iłquel se trouve dans le compte rendu de la Conférence. 